\title{
Dipolar and chain-linking effects on the rheology of grafted chains in a nanopore under shear at different grafting densities
}

Jensen, Morten Østergaard; Mouritsen, Ole G.; Peters, Günther H.J.

Published in:

Physical Review E. Statistical, Nonlinear, and Soft Matter Physics

Link to article, DOI:

10.1103/PhysRevE.64.011507

Publication date:

2001

Document Version

Publisher's PDF, also known as Version of record

Link back to DTU Orbit

Citation (APA):

Jensen, M. Ø., Mouritsen, O. G., \& Peters, G. H. J. (2001). Dipolar and chain-linking effects on the rheology of grafted chains in a nanopore under shear at different grafting densities. Physical Review E. Statistical, Nonlinear, and Soft Matter Physics, 64(1), [011507]. https://doi.org/10.1103/PhysRevE.64.011507

\section{General rights}

Copyright and moral rights for the publications made accessible in the public portal are retained by the authors and/or other copyright owners and it is a condition of accessing publications that users recognise and abide by the legal requirements associated with these rights.

- Users may download and print one copy of any publication from the public portal for the purpose of private study or research.

- You may not further distribute the material or use it for any profit-making activity or commercial gain

- You may freely distribute the URL identifying the publication in the public portal 


\title{
Dipolar and chain-linking effects on the rheology of grafted chains in a nanopore under shear at different grafting densities
}

\author{
Morten $\emptyset$. Jensen, Ole G. Mouritsen, and Günther H. Peters* \\ Membrane and Statistical Physics Group (MEMPHYS), Department of Chemistry, Technical University of Denmark, DTU 207, \\ DK-2800 Lyngby, Denmark
}

(Received 7 August 2000; published 28 June 2001)

\begin{abstract}
Nonequilibrium molecular dynamics simulations are applied to investigate the rheological properties of coplanar nanopore systems of amphiphilic chain molecules with the tails grafted to the walls of the nanopore and with the head-group ends immersed in a solvent inside the nanopore. In particular, the effects of modifying the interaction between the amphiphilic head-groups by repulsive dipolar interactions or directly covalently linking pairs of chains at the head-groups have been studied. Different grafting densities are considered. The chains are modeled by a harmonic bead-spring model, and all particles interact through the repulsive part of a shifted Lennard-Jones potential. Head-group linking is also governed by a bead-spring potential. A harmonic potential models the lattice vibrations of the atomic boundaries. The rheological properties are studied by a shearing process in which the heat generated is conducted away from the system through the walls by applying a Nosé-Hoover thermostat. Computed geometric parameters such as average chain length and average tilt angle indicate reduction in chain flexibility at large dipole moments. Dipolar repulsion is found to broaden the density profiles of the solvent. This effect is opposed by chain linking. For increasing head-group repulsion, the amphiphile-solvent interfaces become less diffusive that leads to systematic variations in viscosities with increasing dipole moments. Friction forces become stronger at large grafting density and for larger dipole moments. The changes in rheological properties for fixed grafting density are essentially governed by the change in the response of the normal pressure to the applied shear field. The velocity gradients depend strongly on the degree of grafting density but appear to be less sensitive to the strength of the interactions between the head groups.
\end{abstract}

DOI: 10.1103/PhysRevE.64.011507

PACS number(s): 83.10.Rs, 68.08.-p, 36.20.Ey

\section{INTRODUCTION}

In the field of material science the ability of fabricating interfaces with designed performance has been utilized in many areas such as the stability of colloids, adhesion, wettability of surfaces, corrosion, and lubrication between surfaces [1-6]. Controlling, optimizing, and designing the physicochemical interfacial properties is a nontrivial task and requires knowledge at the molecular level. In this regard, exploitation of the propensity of organic molecules to self assemble and form supported monolayers is a valuable tool. Among the well-known and highly controlled self-assembled systems are those involving supported monolayers of alkyl thiols. These molecules adsorb onto gold surfaces and form highly oriented, dense, organic monolayers that are remarkably robust and thermodynamically stable $[7,8]$. Similarly, phospholipids may also be grafted to metallic surfaces by using the proper coupling reagents in the grafting step [9]. Furthermore, the supported monolayer formed is easily converted into a bilayer [9]. Formation of self-assembled monolayers involving adsorption to silicon and glass has also been reported [10].

Being able to control the grafting of different organic or biological molecules provides an avenue of introducing a chemical functionality at the free, nongrafted end. By chemically modifying the interfaces, one may in a highly con-

\footnotetext{
*Author to whom correspondence should be addressed.
}

trolled and specific manner promote the desired material performance. Such chemical modifications may be accomplished in various ways. A successful route is the variation of the polarity of the free head group, which was extensively explored by, among others, Bain and Whitesides [7] and Bain, Evall, and Whitesides [8]. These authors introduced different polar head-groups on long chain alkyl thiols end grafted to gold. In turn they observed an interfacial wettability that clearly displayed systematic variation with headgroup polarity; i.e., with the physicochemical state of the designed interface $[7,8]$. Wettability, of course, is only one of several interesting and relevant macroscopic interfacial properties of microscopic origin that one should investigate in order to fully understand nanoscopic organic films and in order to improve their material performance. Mechanical interfacial properties such as friction, to which this study is devoted, is another critical quantity important for a detailed understanding of these films as well as for ultimately improving their performance and persistence in technological applications. Friction that occurs under shear depends strongly on the intermolecular forces transmitted across the interfaces and therefore on the microscopic nature of the interfaces. Clearly, by chemically modifying the interfaces one may be able to manipulate interfacial friction forces. Following the approach in Refs. [7,8], this could be done by varying, e.g., the polarity of the head groups.

Depending on the system, various experimental techniques may measure interfacially transmitted forces. Present experimental methodologies include powerful techniques such as atomic and friction force microscopies, osmotic 
stress measurements, interference reflection spectroscopy, optical tweezers, and micropipette methods [11-14]. Most commonly though, intermolecular forces between coated surfaces are measured using the surface force apparatus (SFA) [15-22]. Measurements using these techniques have revealed intriguing properties of confined liquids, i.e., molecularly thin films in which the thickness of the film is on the nanometer scale. This includes self-assembled monolayers and bilayers as well as structurally less organized systems such as polymeric brushes. Despite the improved resolution of various experimental techniques probing, e.g., friction forces in thin films at the nanoscale level [23], detailed information at the molecular level is still difficult to extract from experimental data. One may therefore argue that insight into the molecular origin of, e.g., shear rheological properties probably is most directly accessed by computational approaches. Along these lines the intrinsic mechanical and geometrical properties of end-grafted monolayer and bilayer systems as well as of polymeric brushes have been subjected to a significant number of computational studies. Molecular dynamics (MD) and Langevin dynamics techniques [24-27] as well as Monte Carlo methods [28,29] have all extensively been applied; see, e.g., [30] and references therein.

Not only from a fundamental point of view but also from the perspective of technological applications (low friction enhances the lifetime of nanoscale equipments) the two most important quantities to be understood when studying molecularly thin films under shear are friction forces and viscosities. To address this issue several studies have been carried out within the framework of mesoscopic modeling, i.e., a coarse graining of an atomic-scale description. Hereby one benefits by being able to approach much longer time scales, typically well within the nanosecond regime, than when using full atomistic modeling. For studies of, e.g., rheological properties of molecularly thin films, the loss of atomic detail is more than well balanced by the ability of approaching longer time scales and thereby providing a solid statistical basis for a reliable computational determination of friction forces and viscosities.

The present study is motivated by the work in Refs. $[7,8]$. We here focus on how mechanical interfacial properties such as friction and viscosity may be affected and controlled by chemical modification of the head-groups of amphiphiles constituting a nanoscopic film. Furthermore, we investigated the rheological properties of the modified interfaces using two different grafting densities, i.e., different amphiphilic coverages of the atomic grafting surface. This study should provide a better understanding at the molecular level of how specific chemical modifications could be applied to construct interfaces that influence and thereby govern macroscopic rheological properties in a desired way.

The molecules applied here are grafted at their tail ends to a solid atomic surface, (i.e., a supported monolayer). The molecules are pairwise unlinked or linked together at their free head-groups which means a chemical cross linking of the amphiphiles in each individual monolayer. The response of the amphiphilic molecules to the applied shear field is analyzed in terms of conformational and mechanical changes. Conformational properties calculated during the

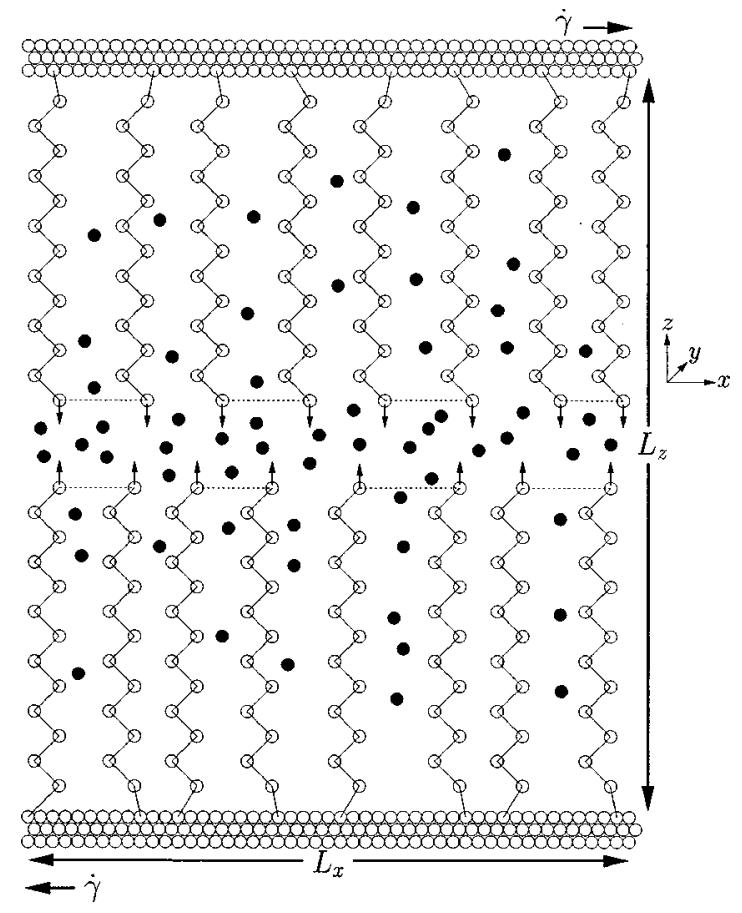

FIG. 1. Schematic representation of the simulation cell. Arrows symbolize dipolar head groups and dashed lines indicate the optional linking of head groups. Solvent particles are symbolized by bold circles.

simulations include average dimensions of the chains, a chain tilt order parameter, and different density profiles. The mechanical response to shear is monitored by calculating the pressure tensor, the friction coefficient, and the viscosity profiles across the nanopore.

In all simulations presented, a constant reduced shear rate of 0.0101 has been applied. This rate was chosen from a previous study which showed that this rate leads to a sufficiently high signal-to-noise ratio and hence to reliable friction and viscosity data [31]. This reduced shear rate corresponds to an experimental shear rate of $10^{-3} \mathrm{~ns}^{-1}$ [32], which is somewhat higher than the shear rates used in SFA experiments $[15-17,19,20,33]$. The systems studied here are related to the systems considered in Refs. [31], [32] in the sense that they are based on similar model potentials. However, only low to intermediate grafting densities $(\leqslant 33 \%)$ were considered and no investigations regarding chemical modification of the interface were done in Refs. [31], [32]. It is exactly this area that is addressed in the present study, the relevance earlier being demonstrated in Refs. [7], [8].

The paper is organized as follows. In Sec. II we describe the model and present the simulation details including the equilibration and sampling procedures. The results are discussed in Sec. III, and finally Sec. IV summarizes the main findings.

\section{MODEL AND SIMULATION DETAILS}

\section{A. Model and potentials}

Figure 1 illustrates the simulation cell that is composed of amphiphilic chains tail-grafted to two atomic boundary walls 
and surrounded by solvent molecules. We shall denote the central solvent region as the pore region and when subdividing the system into discrete slices normal to the $z$ axis, i.e., slices in the $x y$ plane, each slice will be referred to as a slab. Each boundary wall contains 306 boundary atoms. These are arranged in three hexagonally close-packed layers. The arrows displayed at the end of the chain's head groups symbolize point dipoles and the dashed lines indicate optional linking between head groups. Periodic boundary conditions are applied in the $x$ and $y$ directions, whereas in the $z$ direction solvent molecules are confined between the boundary walls. The chosen reduced cell dimensions are $L_{x}^{*}=17.0$, $L_{y}^{*}=5.83$, and $L_{z}^{*}=33.80$ (in units of $\sigma$ ).

The amphiphiles consist of 14 individual units, so-called beads. The intramolecular bead-bead interaction is modeled by a bead-spring model and the solvent is modeled as a pure Lennard-Jones solvent. The lattice vibration of the atoms in the two hexagonally close-packed boundary walls is described by a harmonic potential. All particles in the system interact through the repulsive part of a shifted, truncated Lennard-Jones (LJ) potential [34]

$$
\begin{aligned}
& U_{\mathrm{LJ}}\left(r_{i j}\right) \\
& =\left\{\begin{array}{l}
4 \epsilon_{i j}\left[\left(\frac{\sigma_{i j}}{r_{i j}}\right)^{12}-\left(\frac{\sigma_{i j}}{r_{i j}}\right)^{6}\right]+\epsilon_{i j}, \quad r_{i j} \leqslant 2^{1 / 6} \sigma_{i j} \\
0, \quad r_{i j}>2^{1 / 6} \sigma_{i j} .
\end{array}\right.
\end{aligned}
$$

$\sigma_{i j}$ and $\epsilon_{i j}$ are the collision diameter and well depth of the full LJ potential, respectively. The $\sigma_{i j}$ and $\epsilon_{i j}$ parameters were calculated using the Lorentz-Berthelot rules [34].

The beads in the chains are connected by anharmonic springs described by the potential $[35,36]$

$$
U_{b b}\left(r_{i j}\right)=\left\{\begin{array}{l}
-\frac{1}{2} k_{b b} R_{0}^{2} \ln \left[1-\left(\frac{r_{i j}}{R_{0}}\right)^{2}\right], \quad r_{i j} \leqslant R_{0}, \\
\infty, \quad r_{i j}>R_{0},
\end{array}\right.
$$

where $b b$ denotes bead-bead. The interaction parameters, $k_{b b}=30 \epsilon / \sigma^{2}$ and $R_{0}=1.5 \sigma$, are taken from Ref. [36]. Two chains may be linked together through the head groups of each individual chain. This amphiphilic head-group interaction is also modeled by the potential described by Eqs. (3) and (4).

Additionally, we choose to introduce repulsive scalar dipolar interactions between the head groups

$$
U_{d}\left(r_{i j}\right)=\frac{\mu^{2}}{r_{i j}^{3}},
$$

where identical magnitudes of the dipole moments $\mu$ are assigned to head groups $i$ and $j$.

The $i$ th atom of the atomic boundary is confined to vibrate around the hexagonal lattice point, $\mathbf{r}_{\mathrm{eq}, i}$, through the harmonic potential

$$
U_{\text {wall }}\left(\mathbf{r}_{i}\right)=\frac{1}{2} k_{\text {wall }}\left|\mathbf{r}_{i}-\mathbf{r}_{\mathrm{eq}, i}\right|^{2},
$$

where the force constant $k_{\text {wall }}$ is taken to be $72 \epsilon /\left(2^{1 / 3} \sigma^{2}\right)$ [37]. This yields a harmonic potential well comparable to the full LJ potential. The well depth is sufficiently large to prevent wall atoms from moving out of their equilibrium positions, to allow sufficient heat transfer, and to prevent solvent particles from entering the boundaries.

\section{B. Simulation details}

A series of nonequilibrium MD simulations were performed to explore the effects of dipoles, head-group linking as well as grafting density on the rheology of the amphiphiles. The configurations and simulation times are summarized in Table I. Initial configurations were set up by randomly or periodically grafting amphiphilic molecules to the two atomic boundary walls. Solvent molecules were distributed randomly in the region between the opposing walls (see Fig. 1) and insertion was only allowed in the case of nonoverlapping $\sigma$ 's. Two different grafting densities with respective chain numbers of 34 and 68 were deployed corresponding to grafting densities of 33 and $66 \%$, respectively. In both cases the total density, $\rho^{*}$ was $0.825\left(\rho^{*}\right.$ $\left.=\sigma^{3} N / V\right)$. Increasing the grafting density can be accomplished by maintaining either the total density or the total number of solvent particles. In this study we choose to maintain the total density. Hence an increase in grafting density from 33 to $66 \%$ implies a compensatory reduction in the number of solvent particles.

Furthermore, since we are interested in how friction and viscosity are affected by the underlying subtle interfacial properties we choose to keep the monolayer separation fixed (via $L_{z}$, see Fig. 1) at a distance where the interfaces can be expected to couple through dipolar interactions for both grafting densities. Approaching the limit of uncoupled interfaces is of minor interest from a rheological point of view and is therefore not addressed in this study.

As a starting point for the setup of the chain-linked, i.e., the pairwise head-group linked configurations, a periodic, nonrandom, initial configuration was applied. At first, single amphiphiles were periodically grafted to every third boundary atom along the first row (in the $x$ direction of Fig. 1) in the layer of the boundary wall adjacent to the pore region. Subsequently, the next set of single amphiphiles were grafted with the same period to the second row of boundary atoms in this layer. Additionally, the constraint was imposed that the grafting points of the second row has to lead to bond distances, $\left(r_{i j}\right)$, to the amphiphiles present in the first row so that $r_{i j}^{*} \leqslant 1.5 \forall i, j$. The indices $i, j$ label the pairwise bonded head groups. A grafting density of $33 \%$ was used.

In the simulations with dipolar head-group interactions, different reduced dipole strengths ranging from $\mu^{*}=1.1$ to 25.0 were applied $\left[\mu^{*}=\mu /\left(\sigma^{3} \epsilon\right)^{1 / 2}\right.$, where $\mu$ is given in Debye]. The largest dipole strength resembles dipole moments of zwitterionic phospholipids [38]. For the purpose of direct comparison between linked and nonlinked systems when dipolar interactions are introduced, we choose to assign one dipole moment to each of the head groups of the linked configurations. Accordingly the same number of dipoles and the same total dipole moment in each amphiphilic layer are maintained for all values of $\mu$ applied and regardless of linking status. This choice necessarily implies that our 
TABLE I. Summary of all systems simulated. The abbreviations are as follows: Conf. denotes configuration; $P$ and $R$ indicate periodic and random chain assignment, respectively; $L$ indicates head-group linking; Cov. refers to grafting density expressed in $\%$ of the total number of particles present in the layer of the boundary surface adjacent to the pore region; $\mu^{*}$ is the reduced dipole moment; and $\dot{\gamma}^{*}$, is the reduced shear rate (see text). The length of the trajectories, $t$, is given in nanoseconds.

\begin{tabular}{|c|c|c|c|c|c|c|c|c|c|}
\hline Conf. & Cov. & $\mu^{*}$ & $\dot{\gamma}^{*}$ & $t$ & Conf. & Cov. & $\mu^{*}$ & $\dot{\gamma}^{*}$ & $t$ \\
\hline$R$ & 33 & 0.0 & 0.0 & 1.25 & $R$ & 66 & 0.0 & 0.0 & 1.25 \\
\hline$R$ & 33 & 0.0 & 0.0101 & 1.25 & $R$ & 66 & 0.0 & 0.0101 & 1.25 \\
\hline$R$ & 33 & 1.1 & 0.0 & 1.25 & $R$ & 66 & 1.0 & 0.0 & 1.25 \\
\hline$R$ & 33 & 1.1 & 0.0101 & 1.25 & $R$ & 66 & 1.1 & 0.0101 & 1.25 \\
\hline$R$ & 33 & 2.2 & 0.0 & 1.25 & $R$ & 66 & 2.2 & 0.0 & 1.25 \\
\hline$R$ & 33 & 2.2 & 0.0101 & 1.25 & $R$ & 66 & 2.2 & 0.0101 & 1.25 \\
\hline$R$ & 33 & 6.0 & 0.0 & 1.25 & $R$ & 66 & 6.0 & 0.0 & 1.25 \\
\hline$R$ & 33 & 6.0 & 0.0101 & 1.25 & $R$ & 66 & 6.0 & 0.0101 & 1.25 \\
\hline$R$ & 33 & 12.5 & 0.0 & 1.25 & $R$ & 66 & 12.5 & 0.0 & 1.25 \\
\hline$R$ & 33 & 12.5 & 0.0101 & 1.25 & $R$ & 66 & 12.5 & 0.0101 & 1.25 \\
\hline$R$ & 33 & 25.0 & 0.0 & 2.50 & $R$ & 66 & 25.0 & 0.0 & 2.50 \\
\hline$R$ & 33 & 25.0 & 0.0101 & 2.50 & $R$ & 66 & 25.0 & 0.0101 & 2.50 \\
\hline$P$ & 33 & 0.0 & 0.0 & 1.25 & $P, L$ & 33 & 0.0 & 0.0 & 1.25 \\
\hline$P$ & 33 & 0.0 & 0.0101 & 1.25 & $P, L$ & 33 & 0.0 & 0.0101 & 1.25 \\
\hline$P$ & 33 & 1.1 & 0.0 & 1.25 & $P, L$ & 33 & 1.1 & 0.0 & 1.25 \\
\hline$P$ & 33 & 1.1 & 0.0101 & 1.25 & $P, L$ & 33 & 1.1 & 0.0101 & 1.25 \\
\hline$P$ & 33 & 2.2 & 0.0 & 1.25 & $P, L$ & 33 & 2.2 & 0.0 & 1.25 \\
\hline$P$ & 33 & 2.2 & 0.0101 & 1.25 & $P, L$ & 33 & 2.2 & 0.0101 & 1.25 \\
\hline$P$ & 33 & 6.0 & 0.0 & 1.25 & $P, L$ & 33 & 6.0 & 0.0 & 1.25 \\
\hline$P$ & 33 & 6.0 & 0.0101 & 1.25 & $P, L$ & 33 & 6.0 & 0.0101 & 1.25 \\
\hline$P$ & 33 & 12.5 & 0.0 & 1.25 & $P, L$ & 33 & 12.5 & 0.0 & 1.25 \\
\hline$P$ & 33 & 12.5 & 0.0101 & 1.25 & $P, L$ & 33 & 12.5 & 0.0101 & 1.25 \\
\hline$P$ & 33 & 25.0 & 0.0 & 1.25 & $P, L$ & 33 & 25.0 & 0.0 & 1.25 \\
\hline$P$ & 33 & 25.0 & 0.0101 & 1.25 & $P, L$ & 33 & 25.0 & 0.0101 & 1.25 \\
\hline
\end{tabular}

model amphiphiles somewhat artificially have two dipolar interaction sites.

The shear field was induced by sliding the boundary walls in opposing directions (see Fig. 1). Sliding the atomic walls in opposite directions yields the overall displacement

$$
\Delta x= \pm \frac{1}{2} \dot{x} L_{z} \Delta t
$$

A reduced shear rate $\dot{\gamma}^{*}$ of 0.0101 was applied [where $\dot{\gamma}^{*}$ $=\dot{\gamma}\left(M \sigma^{2} / \epsilon\right)^{1 / 2}$ is the shear rate in reduced units, $M$ is the mass of a bead, and $\sigma$ and $\epsilon$ are the usual parameters of the LJ potential defined in Eqs. (1) and (2)]. As stated in Sec. I, this value was based on previous studies, where it was observed that 0.0101 is the lowest possible shear rate that yields reliable friction and viscosity data and at the same time approaches experimental shear rates $[31,32]$. The simulations were carried out in the $N V T$ ensemble and performed at a reduced temperature of $T^{*}=2.083\left(T^{*}=k_{B} T / \epsilon\right)$ by coupling the wall atoms to a Nosé-Hoover heat bath. The neighboring list was updated every tenth time step. Integration of the equations of motion were carried out using the leap-frog algorithm [39]. As usual $\tau=t\left(M \sigma^{2} / \epsilon\right)^{-1 / 2}$ is the reduced time expressed in the Lennard-Jones parameters. The systems were initially equilibrated for $5 \times 10^{4}$ time steps $(\Delta \tau$
$=0.0025)$ or $1 \times 10^{5}$ time steps $(\Delta \tau=0.00125)$ followed by sampling periods of $5 \times 10^{5}(\Delta \tau=0.0025)$ or $1 \times 10^{6}-2$ $\times 10^{6}(\Delta \tau=0.00125)$ time steps resulting in sampling times of 1.25-2.5 ns. The smaller time step was required for the simulations with the largest dipole moment to ensure a correct integration of the equations of motion. This was initially checked by monitoring energy conservation of the simulations in the $N V E$ ensemble with different dipole moments and no applied shear rate. For all systems in question the observed drift in energy was within the accepted and expected range using the integrator above [39]. For systems simulated with shear, the equilibrated configuration of the corresponding system without shear was used as the starting configuration for the equilibration.

\section{RESULTS AND DISCUSSION}

Our presentation of the results is subdivided into two sections. In the first section we discuss the geometric changes of the grafted amphiphiles induced by the shear, while the second section contains thermodynamic and viscosity data obtained in the presence of shear. Unless explicitly stated, we limit our discussion concerning the nonlinked configurations 


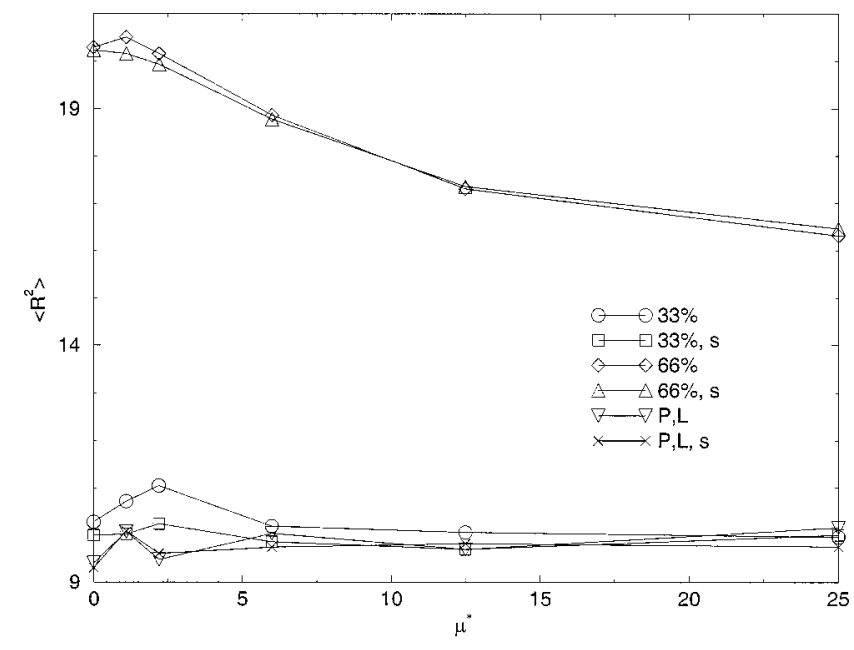

FIG. 2. Squared radius of gyration $\left\langle R^{2}\right\rangle$ as a function of reduced dipole moment $\mu^{*}$, calculated according to Eqs. (8)-(10) with and without shear applied. Shear is indicated by an $s$ in the inset. Numbers in the inset refer to grafting densities of random (nonlinked) configurations (see Table I caption). $P, L$ denotes the periodic, linked configuration.

at low grafting density to the random setup, i.e., $R, 33 \%$, of Table I.

\section{A. Geometric quantities}

Molecular information providing insight into the averaged configurational state of the amphiphiles in the presence and absence of shear may be gained by analyzing various geometric parameters such as chain tilting, squared end-to-end distances, and radii of gyration. These parameters are in particular used to investigate the effect of introducing dipoles and chain linkages at the amphiphilic head groups. All geometric quantities reported in this subsection are given in reduced units, i.e., all lengths are reduced by a factor of $1 / \sigma$. For convenience, we have left out the asterisk, indicating the use of reduced Lennard-Jones units, in these particular length parameters.

Averaging over time and chains the mean square radius of gyration may be computed as

$$
\left\langle R^{2}\right\rangle=\left\langle R_{\|}^{2}\right\rangle+\left\langle R_{\perp}^{2}\right\rangle
$$

with parallel, $\|$, and normal, $\perp$, components given by [40]

$$
\begin{gathered}
\left\langle R_{\|}^{2}\right\rangle=\frac{1}{K L} \sum_{k=1}^{K} \sum_{l \in k}^{L}\left\langle\left(x_{l}-x_{k}\right)^{2}+\left(y_{l}-y_{k}\right)^{2}\right\rangle \\
\left\langle R_{\perp}^{2}\right\rangle=\frac{1}{K L} \sum_{k=1}^{K} \sum_{l \in k}^{L}\left\langle\left(z_{l}-z_{k}\right)^{2}\right\rangle .
\end{gathered}
$$

In Eqs. (9) and (10) $\left(x_{k}, y_{k}, z_{k}\right)$ is the center of mass of the $k$ th chain and the inner summation runs over the number of beads belonging to the $k$ th chain. $\langle\cdots\rangle$ denotes a time average.

The squared radius of gyration as a function of $\mu^{*}$ is shown in Fig. 2 and the parallel component of $\left\langle R^{2}\right\rangle$ is dis-

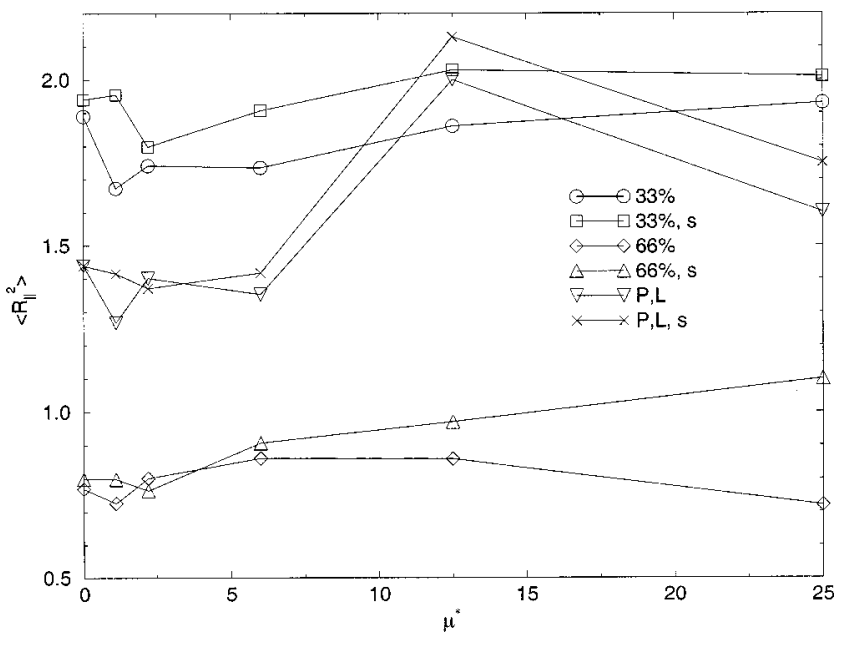

FIG. 3. Squared parallel component of radius of gyration, $\left\langle R_{\|}^{2}\right\rangle$, as a function of reduced dipole moment $\mu^{*}$, calculated according to Eq. (9) with and without shear applied. See Fig. 2 caption for more details.

played in Fig. 3. By introducing dipolar head-group interactions we observe two opposing effects on the conformation of the chains. At low grafting density and low dipole strength $\left(\mu^{*} \leqslant 2.2\right)$, the repulsion between the head groups located within the same layers is dominant. These repulsive interactions are sufficiently strong to cause an extension of the chains and hence an inherent ordering within the layer. As $\mu^{*}$ is further increased, the dipolar repulsive forces between the more distant head groups of the two opposing amphiphilic layers become significant resulting in a shortening of the chains. For $\mu^{*}=0.0,\left\langle R^{2}\right\rangle$ is lower for the linked configurations than for the corresponding nonlinked chains at $33 \%$ density. We may therefore conclude that chain linking leads to a shortening of the amphiphiles in absence of dipoles. For $\mu^{*}$ being nonzero the linked amphiphiles straighten slightly and for $\mu^{*} \geqslant 6.6$ linked and nonlinked chains have approximately the same values of $\left\langle R^{2}\right\rangle$.

At low grafting density the dependence of $\left\langle R^{2}\right\rangle$ on $\mu^{*}$ is only moderate, whereas much stronger effect of $\mu^{*}$ on $\left\langle R^{2}\right\rangle$ is observed at high grafting density. When comparing results for both grafting densities and same value of $\mu^{*},\left\langle R^{2}\right\rangle$ is significantly larger at high grafting density than at low density. This reflects the closer chain packing for the denser system and consequently less conformational freedom of the chains. At both grafting densities $\left\langle R^{2}\right\rangle$ decreases when the dipolar repulsion becomes stronger. This observation is, however, most significant at 66\%. At this grafting density the head groups of opposing amphiphilic layers are more closely located than at $33 \%$ and hence the decrease in $\left\langle R^{2}\right\rangle$ at $66 \%$ is more pronounced due to the stronger dipolar interactions across the pore than at $33 \%$.

The dependence of the grafting density on the chain packing is also reflected in the parallel component of $\left\langle R^{2}\right\rangle$ shown in Fig. 3. $\left\langle R_{\|}^{2}\right\rangle$ is notably smaller at high grafting density than at low density, reflecting denser packing and hence a decrease in conformational freedom of the chains. When no shear is applied we note that small dipole moments lower $\left\langle R_{\|}^{2}\right\rangle$, since weak repulsion overall straightens the chains ir- 
respective of the degree of grafting density. Under shear, the chains start to tilt, and consequently $\left\langle R_{\|}^{2}\right\rangle$ increases. The dependence of $\left\langle R_{\|}^{2}\right\rangle$ on the shear field becomes stronger when $\mu^{*}$ is nonzero, indicating that chain tilting under shear is reinforced by head-group repulsion. At low grafting density the chains have more configurational freedom to fold and entangle resulting in a larger $\left\langle R_{\|}^{2}\right\rangle$ regardless of the presence of shear. When no dipoles are present, chain linking reduces $\left\langle R_{\|}^{2}\right\rangle$ and a significant maximum is displayed around $\mu^{*}$ $=12.5$.

In all systems studied the normal component $\left\langle R_{\perp}^{2}\right\rangle$ has a maximum at $\mu^{*} \leqslant 2.2$ (data not shown). Therefore shear in general causes the amphiphiles to shorten slightly in the presence of dipoles. According to Fig. $3\left\langle R_{\|}^{2}\right\rangle$ does change significantly under shear but the numerical change is an order of magnitude smaller than for $\left\langle R_{\perp}^{2}\right\rangle$ (data not shown). Hence the overall change in radius of gyration induced by shear (Fig. 2) is predominantly determined by changes in $\left\langle R_{\perp}^{2}\right\rangle$.

Similar characteristics are observed for the squared endto-end distance $\left\langle R_{\mathrm{ee}}^{2}\right\rangle$ of the amphiphiles (data not shown). We find that $\left\langle R_{\text {ee }}^{2}\right\rangle$ initially increases for weak dipolar repulsion and then becomes lower with increasing dipolar repulsion. Shear causes $\left\langle R_{\text {ee }}^{2}\right\rangle$ to be slightly reduced. The largest structural response of the chains to an increased dipole moment is observed in the simulations with high grafting density. Again this is rationalized by the close contacts between the head groups of the opposing amphiphilic layers. In the absence of dipoles, $\left\langle R_{\mathrm{ee}}^{2}\right\rangle$ is lowered by chain linking; however dipolar repulsions between head groups in the same layer cause the linked chains to straighten and thereby $\left\langle R_{\mathrm{ee}}^{2}\right\rangle$ converges toward the same values as those obtained for the nonlinked counterparts.

In order to gain more specific information on the degree of chain tilting under shear, we define a tilt order parameter as the configurationally time averaged quantity

$$
1-\langle O\rangle=1-\frac{1}{K} \sum_{k=1}^{K}\left\langle P_{2}\left(\cos \theta_{k}\right)\right\rangle=\frac{1}{2 K} \sum_{k=1}^{K}\left\langle 3 \cos ^{2} \theta_{k}-1\right\rangle,
$$

where $P_{2}$ is the second Legendre polynomium. $\theta_{k}$ is the angle between the surface normal, ( $\hat{\mathbf{z}}$, see Fig. 1 ) and the principal axis of the inertia tensor having the smallest eigenvector, i.e., the long axis along which the amphiphile possesses the largest moment of inertia. The summation runs over all $K$ chains regardless of linking status. Figure 4 displays the tilt order parameter as a function of $\mu^{*}$. Values for $1-\langle O\rangle$ of 0 and 1 correspond to the fact that the average orientations of the amphiphiles are perpendicular and parallel to $\hat{\mathbf{z}}$, respectively.

At high grafting density, $1-\langle O\rangle$ is significantly lower than observed at low density. This again reflects the denser chain packing. As expected, in all systems, the induced shear causes an increase in chain tilting. At low grafting density and in absence of dipoles, chain linking prevents tilting significantly except for $\mu^{*}=12.5$ where we also observed that $\left\langle R_{\|}^{2}\right\rangle$ had a pronounced maximum (Fig. 3). Interestingly for

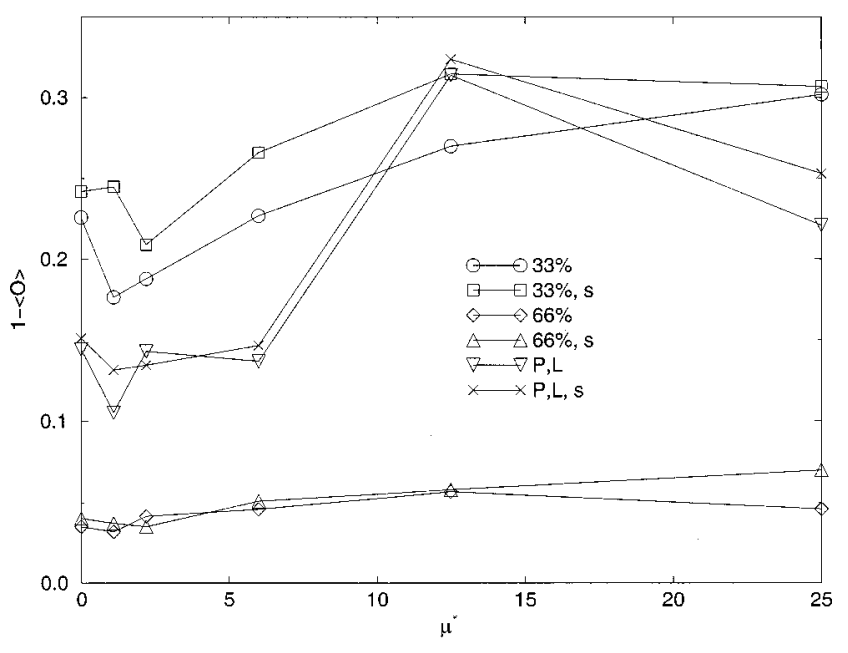

FIG. 4. Tilt order parameter $1-\langle O\rangle$ as a function of reduced dipole moment $\mu^{*}$, calculated according to Eq. (11) with and without shear applied. See Fig. 2 caption for more details.

small dipole moments $\left(\mu^{*} \leqslant 2.2\right)$, the weak repulsion decreases the order parameter in all systems. By increasing the dipole moment further, $1-\langle O\rangle$ increases. For the linked configuration this increase leads to a maximum value of 1 $-\langle O\rangle$ around $\mu^{*}=12.5$. The initial decrease in $1-\langle O\rangle$ is reflecting the initial straightening of the chains that occurred when weak dipolar repulsion was switched on (see Fig. 2). At high grafting density the variation in $1-\langle O\rangle$ with increasing dipole moment is less pronounced than observed at $33 \%$. This essentially shows that the increased ordering is due to the higher grafting density.

\section{B. Shear rheology}

Structural ordering in the system can be deduced from histogram sampling of the particle number density across the system. The density in the slab $z_{n}$ was for this purpose calculated as [41]

$$
\rho\left(z_{n}\right)=\frac{1}{L_{x} L_{y}} \sum_{i=1}^{N}\left\langle H_{n}\left(z_{i}\right)\right\rangle,
$$

where the step function is given as

$$
H_{n}\left(z_{i}\right)= \begin{cases}1 & \text { for } z_{n}-\frac{\Delta z}{2}<z_{i}<z_{n}+\frac{\Delta z}{2} \\ 0, & \text { otherwise. }\end{cases}
$$

In Eq. (12) the summation runs over all particles present in the system. Sampling was made at every tenth time step. Figure 5 displays the solvent $\rho^{*}\left(z^{*}\right)_{\text {so }}$ and total number density profiles $\rho^{*}\left(z^{*}\right)_{\text {to }}$ at different dipole moments. At low grafting density a continuous widening of the distribution of the solvent particles is seen when increasing the dipole moment up to $\mu^{*}=12.5$ [Fig. 5(a)]. Increasing $\mu^{*}$ further to 25.0 only affects $\rho^{*}\left(z^{*}\right)$ marginally resulting in almost identical density curves for $\mu^{*}=12.5$ and $\mu^{*}=25.0$. The dipolar repulsion between the head groups apparently creates vacan- 


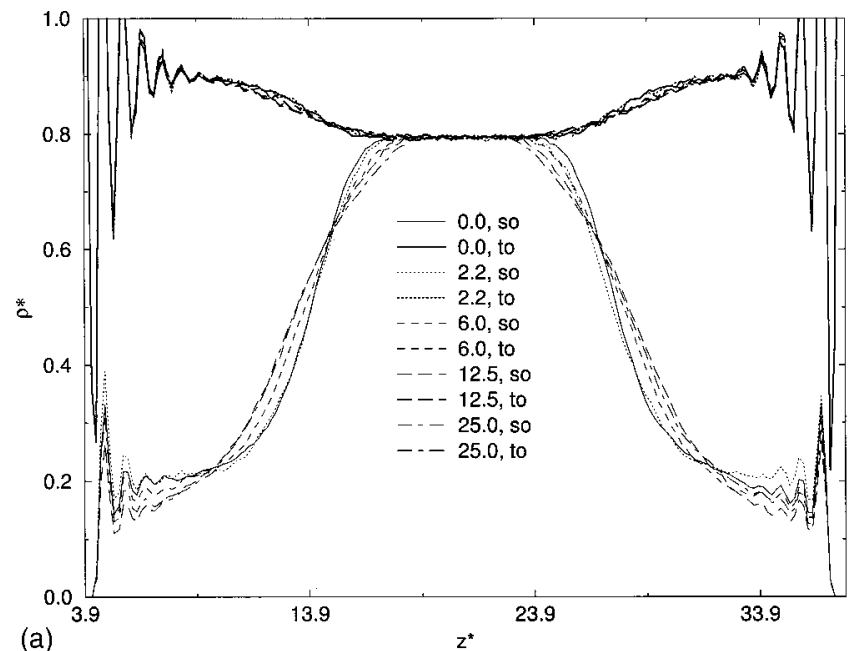

(a)

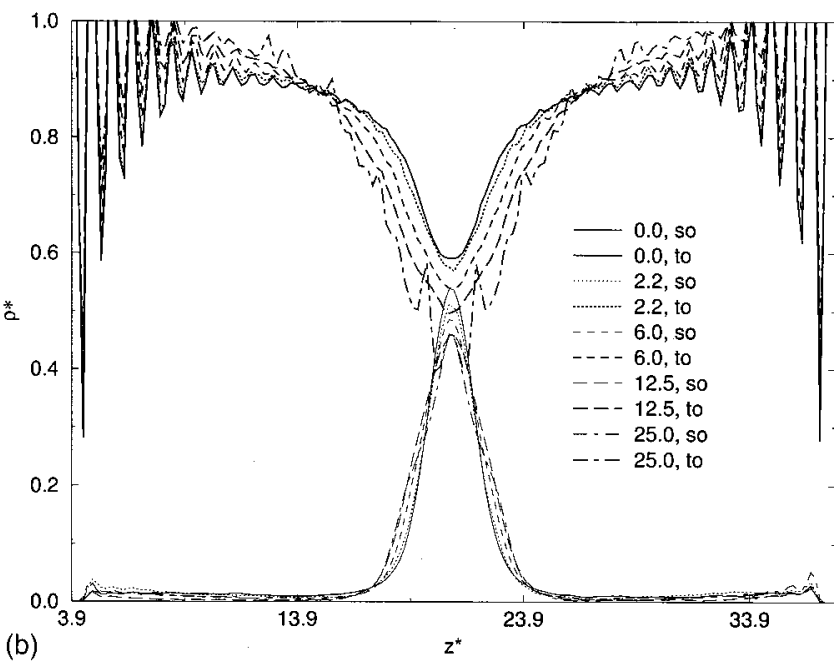

(b)

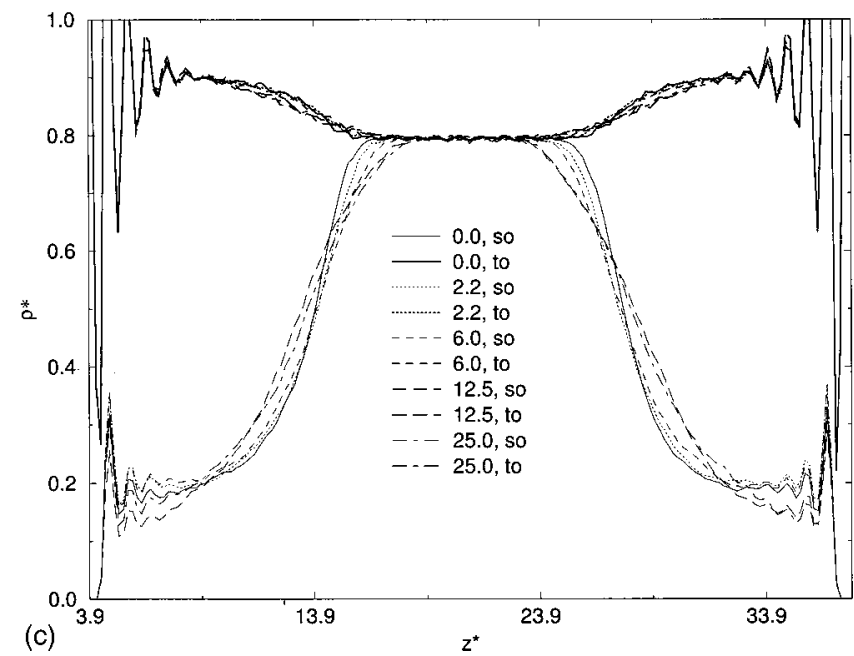

FIG. 5. Density profiles $\rho\left(z^{*}\right)$, across the nanopore for different reduced dipole moments $\mu^{*}$. The lower profiles are solvent (so) densities, upper profiles are total (to) densities. The profiles are calculated according to Eq. (12) with shear applied for the random configurations at $33 \%$ (a) and $66 \%$ (b) grafting density and for the periodic, linked configuration, $P, L$ (c). For clarity, the profiles for $\mu^{*}=1.1$ have been omitted and only the slab densities between the boundaries are shown, i.e., for $3.89 \leqslant z^{*}<37.69$. cies in between the chains in which the solvent particles can penetrate. This in turn yields a solvent distribution extending further into the grafted chain regions. The marginal differences observed in solvent distributions between $\mu^{*}=12.5$ and 25.0 indicate that essentially no more vacancies are generated in this range.

As also observed by the geometrical quantities, the largest dipolar effect on the structural properties in the system is seen at a high grafting density that is clearly demonstrated by the density profiles at $66 \%$ [Fig. 5(b)]. Some of the features of these profiles can be explained in terms of the constant total density $\left(\rho^{*}=0.825\right)$. As discussed before, high grafting density implies removal of solvent particles in order to maintain constant total density. In turn solvent and total densities both drop significantly in the pore (solvent) region. Again we find that increasing dipolar repulsion promotes solvent distribution toward the boundaries and solvent depletion in the pore region. The gap present in the profiles for $\mu^{*} \leqslant 6.0$ between total and solvent densities implies that the total density in the central region is not constituted by the solvent alone but also by the chains. However, for $\mu^{*}=12.5$ and $\mu^{*}=25.0$ repulsion between the head groups located in opposing amphiphilic layers becomes so significant that the gap is closed by solvent molecules. Density fluctuations become larger with an increasing chain density. Overall, the pronounced roughening of the density curves reflects the increased stiffness of the system.

At low density, chain linking [Fig. 5(c)] causes the solvent to localize slightly more in the pore region since headgroup linking opposes dipolar repulsion between the two linked, dipolar head-groups. Compared to the corresponding unlinked configurations, the solvent-accessible space in between the linked chains is reduced, and the solvent consequently has to localize more toward the center of the pore in these systems. Interestingly, by increasing the dipole strength from $\mu^{*}=12.5$ to $\mu^{*}=25.0$ the profile for the solvent is slightly narrowed. This suggests that the chain-linking bond stretches until the dipolar repulsion from neighboring chains becomes so large $\left(\mu^{*}=25.0\right)$ that the bond again is forced to shorten. It should be recalled that strong nonmonotonous behavior was also observed for the linked configuration for $\mu^{*}=12.5$ in the plots of $\left\langle R_{\|}^{2}\right\rangle$ and $1-\langle O\rangle$ in Figs. 3 and 4 , respectively.

Turning to the thermodynamic response quantities we

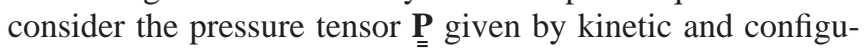
rational contributions [39]

$$
\underline{\underline{P}}=\frac{1}{V}\left(\sum_{i=1}^{N} \frac{\left|\mathbf{p}_{i}\right|^{2}}{m_{i}} \underline{\underline{\mathbf{I}}}+\sum_{i<j}^{N}\left\langle\mathbf{r}_{i j} \mathbf{F}_{i j}^{\dagger}\right\rangle\right) .
$$

In Eq. (13) $\mathbf{p}_{i}$ is the linear momentum of particle $i$ of mass $m_{i}$. I is the Cartesian unit tensor, $\dagger$ indicates a transposed column vector entity, and $\langle\cdots\rangle$ denotes time average. In absence of shear, $\mathbf{P}$ is diagonal as required by mechanical equilibrium under isotropic conditions [42]. Applying a shear field along the $\pm x$ direction (see Fig. 1) breaks the symmetry of the system, and the mechanical response function $\underline{\underline{\mathbf{P}}}$ is no longer diagonal. From the off-diagonal response components we can calculate the viscosities and friction coefficients. In 


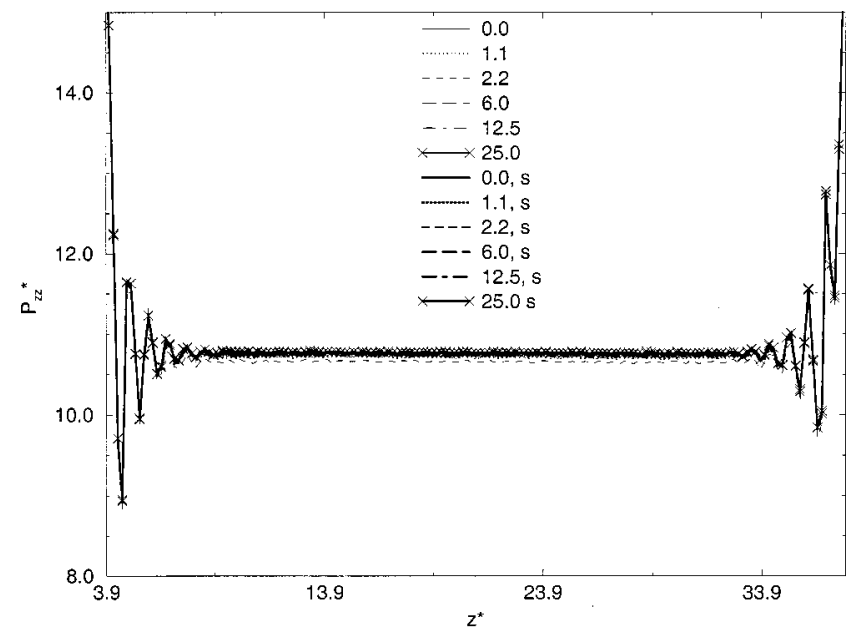

FIG. 6. Normal pressure profiles $P_{z z}^{*}\left(z^{*}\right)\left(P_{z z}^{*}=P_{z z} \sigma^{3} / \epsilon\right)$ for the $R, 33 \%$ configuration across the nanopore for different reduced dipole moments $\mu^{*}$ (given in the inset). These are obtained according to Eq. (14) with and without shear applied. Shear is indicated by an $s$ in the inset. Only the slab pressures between the boundaries are shown, i.e., for $3.89 \leqslant z^{*}<37.69$.

the simulations the pressure tensor is calculated from the Irving-Kirkwood expression [43]

$$
\begin{aligned}
\underline{\underline{\mathbf{P}}}(z)= & \rho(z) k_{B} T \underline{\underline{\mathbf{I}}}-\frac{1}{L_{x} L_{y}} \\
& \times \sum_{i<j}^{N}\left\langle\mathbf{r}_{i j} O_{i j} \nabla_{\mathbf{r}_{i j}}^{\dagger} U\left(r_{i j}\right) \frac{\theta\left(\frac{z-z_{i}}{z_{i j}}\right) \theta\left(\frac{z_{j}-z}{z_{i j}}\right)}{\left|z_{i j}\right|}\right\rangle,
\end{aligned}
$$

where $\underline{\underline{I}}$ again is the diagonal unit tensor, $O_{i j}$ is a differential operator, $\langle\cdots\rangle$ denotes an NVT ensemble average, and $\theta(\cdots)$ is a unit step function, which is 1 for $(\cdots)>0$ and 0 otherwise. The kinetic contribution was obtained through the momentum exchange across the area $A$, which is given by $\Delta p /(\Delta t A)$ as discussed in Ref. [42]. This contribution was evaluated at every time step, whereas the configurational part was sampled at every tenth time step.

Normal pressures, $\left\langle P_{N}^{*}\left(z^{*}\right)\right\rangle=\left\langle P_{z z}^{*}\left(z^{*}\right)\right\rangle$ [see Fig. 1 and Eq. (14)], are plotted in Figs. 6 and 7. $\left\langle P_{N}^{*}\left(z^{*}\right)\right\rangle$ is essentially constant over the whole system. The strong fluctuations that occur at the boundaries (partly shown) may be caused by neglecting higher-order terms in a series expansion of the differential operator $O_{i j}$ in the configurational part of Eq. (14) $[44,45]$. At low grafting density, the values of the normal pressures are hardly affected by the magnitude of $\mu^{*}$. In contrast, at high density, $\left\langle P_{N}^{*}\left(z^{*}\right)\right\rangle$ increases significantly when increasing the strength of the repulsive dipolar potential [Eq. (5)]. The profile for $\mu^{*}=1.1$ obtained in absence of shear is the only exception. Here $\left\langle P_{N}^{*}\left(z^{*}\right)\right\rangle$ is actually lower than the corresponding profile obtained for $\mu^{*}=0.0$. Apparently, the structural ordering of the chains that was characteristic of the smallest dipolar repulsion only (see Figs. 2-4 and Sec. III A), implies that the amphiphiles in absence of shear and for $\mu^{*}=1.1$ are able to adopt a configuration that

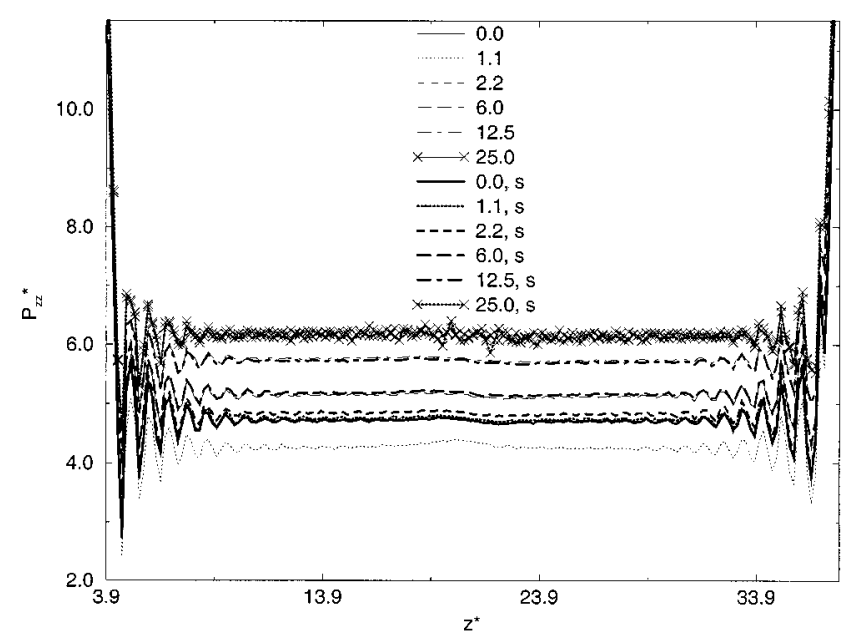

FIG. 7. Normal pressure profiles $P_{z z}^{*}\left(z^{*}\right)$ for the $R, 66 \%$ configuration across the nanopore for different reduced dipole moments, and with and without shear applied. See Fig. 6 caption for more details.

overall causes a lowering of $\left\langle P_{N}^{*}\left(z^{*}\right)\right\rangle$ relative to the corresponding configuration for $\mu^{*}=0.0$.

The general increase in normal pressures with increasing dipole moment can be ascribed to a larger configurational contribution to $\left\langle P_{N}^{*}\left(z^{*}\right)\right\rangle$; see Eqs. (5) and (14). The profiles for the linked configurations (data not shown) are essentially identical to the profiles obtained for low grafting density (Fig. 6).

The off-diagonal response component, $\left\langle P_{x z}^{*}\left(z^{*}\right)\right\rangle$, is shown in Figs. 8 and 9. In the absence of shear it fluctuates around zero as dictated by symmetry and mechanical equilibrium constraints $[43,46]$. Imposing shear leads to profiles of nonzero values. The values become lower with increasing

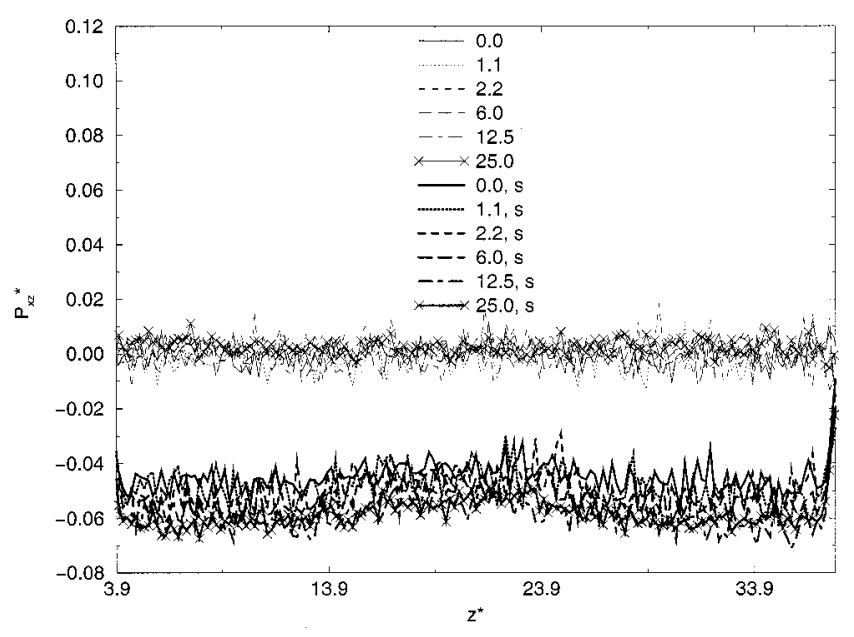

FIG. 8. Off-diagonal pressure profiles $P_{x z}^{*}\left(z^{*}\right) \quad\left(P_{x z}^{*}\right.$ $\left.=P_{x z} \sigma^{3} / \epsilon\right)$ for the $R, 33 \%$ configuration across the nanopore for different reduced dipole moments. These profiles are obtained according to Eq. (14) with and without shear applied. The six lower curves (in bold font) are in the presence of shear (as also indicated by an $s$ in the inset). Only the slab pressures between the boundaries are shown, i.e., $3.89 \leqslant z^{*}<37.69$. 


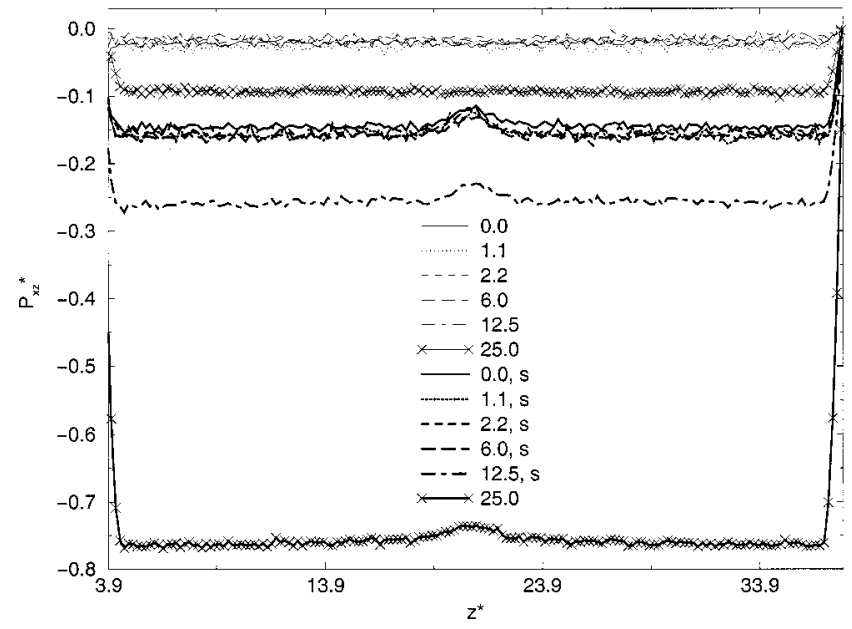

FIG. 9. Off-diagonal pressure profiles $P_{x z}^{*}\left(z^{*}\right)$ for the $R, 66 \%$ configuration across the nanopore for different reduced dipole moments and with and without shear applied. See Fig. 8 caption for more details.

dipole moments due to the increased configurational contribution to $\left\langle P_{x z}^{*}\left(z^{*}\right)\right\rangle$; see Eqs. (5) and (14). For the lowest dipole moments, only small decreases in $\left\langle P_{x z}^{*}\left(z^{*}\right)\right\rangle$ are observed, whereas increased dipolar repulsion influences $\left\langle P_{x z}^{*}\left(z^{*}\right)\right\rangle$ more strongly. Ignoring fluctuations, $\left\langle P_{x z}^{*}\left(z^{*}\right)\right\rangle$ is seen to be constant over the amphiphilic region. This region can be defined as the region that extends approximately $10 \sigma$ and $15 \sigma$ from the atomic boundaries toward the center of the slab for the 33\% (Fig. 8) and 66\% (Fig. 9) grafting densities, respectively. The range of the amphiphilic region over which $\left\langle P_{x z}^{*}\left(z^{*}\right)\right\rangle$ is essentially constant is in agreement with the averaged chain lengths, see, e.g., Fig. 2. Across the solvent region, i.e., across the central part of the pore, we find a noticeable increase in $\left\langle P_{x z}^{*}\left(z^{*}\right)\right\rangle$. This observation is most pronounced for $66 \%$ coverage where the increase roughly occurs over $5 \sigma$. For $33 \%$ coverage we also observe an increase which, however, is less pronounced and occurs over a region of approximately $10 \sigma$. These characteristics of the off-diagonal pressure profiles reflect the differences in the broadness of the solvent distributions obtained for the two different grafting densities (see Fig. 5). The linked configurations essentially have pressure profiles almost identical to their unlinked counterparts in Fig. 8 (data not shown).

The shear viscosity profiles are obtained across the slab through the instantaneous shear rate, $\dot{x}(z)$, given by the velocity gradient, $d \dot{x}(z) / d z$, and through the off-diagonal response component, $\left\langle P_{x z}(z)\right\rangle$, as [47]

$$
\eta(z)=\frac{-\left\langle P_{x z}(z)\right\rangle}{\dot{x}(z)} .
$$

These profiles are shown in Fig. 10. At low grafting density we find almost no change in viscosity in the very central pore region, i.e., at the minimum of the curves in Fig. 10. At large grafting densities, the shear viscosity in the central pore increases approximately by a factor of 2 when $\mu^{*}$ is increased from the smaller $\left(0.0 \leqslant \mu^{*}<6.0\right)$ to the largest dipolar strengths $\left(\mu^{*}=12.5-25.0\right)$; see Fig. 10(b). This is caused by the larger off-diagonal pressure component, since the velocity profile, as shown in the inset, is less dependent on the dipolar strength.

In general, we find that dipolarization of the amphiphilic head groups has a pronounced effect by causing significantly higher viscosities at the interface i.e., the interface becomes much less diffusive. Minor difference in viscosity profiles are noted for dipole strengths of $\mu^{*}=12.5$ and $\mu^{*}=25.0$ at both grafting densities. We note in particular that the pore region of low viscosity clearly narrows when dipolar head groups are present. This holds for all systems studied. The interfacial flow resistance also increases dramatically with increasing chain stiffness, as dictated by denser packing and reflected by the more narrow viscosity profiles obtained at $66 \%$ than at $33 \%$.

When no shear is applied the velocity profiles $\dot{x}^{*}\left(z^{*}\right)$ are zero through the whole slab (data not shown). Displacing the boundary walls in the $\pm x$ directions [see Fig. 1 and Eq. (7)] leads to positive and negative flow velocities in each half of the pore region as seen in the insets of Fig. 10. In contrast, $\dot{x}^{*}\left(z^{*}\right)$ remains constant in the two amphiphilic regions. As expected the tail-grafted amphiphiles have to move with the walls under the applied shear. The chains therefore behave as though they were displaced into the solvent region. This displacement is equal to the hydrodynamic radius of the layer as discussed in [31]. The longitudinal movement of the amphiphiles in the shearing process induces the geometrical changes presented in Figs. 2-4 and are discussed earlier. At low grafting density, $\dot{x}^{*}\left(z^{*}\right)$ becomes steeper across the pore when the head-group repulsion increases and hence the system becomes stiffer. Clearly, $\dot{x}^{*}\left(z^{*}\right)$ is sensitive to the increased stiffness of the amphiphilic system at low coverage. At high grafting density we observe significantly steeper velocity profiles. This is a combined consequence of the fewer solvent particles present (the solvent flow now spans a more narrow region, see Fig. 5) and of the elongation of the amphiphiles (their hydrodynamic radius is larger at $66 \%$ than at $33 \%$, see Fig. 2). However, $\dot{x}^{*}\left(z^{*}\right)$ is only marginally affected by increasing dipolar repulsion that is due to the denser chain packing. Finally, we find that chain linking in general has little influence on $\dot{x}^{*}\left(z^{*}\right)$. Linked and unlinked chains practically yield identical velocity and viscosity profiles.

The dimensionless friction coefficient for the solvent can be obtained through the so-called shear stress component, $-\left\langle P_{x z}\right\rangle$, (i.e., the negative of the response of the normal pressure to the applied shear in the $x$ direction) and the normal pressure $\left\langle P_{z z}\right\rangle$ [47]

$$
\xi\left(z=z_{0}\right)=\frac{-\left\langle P_{x z}\left(z=z_{0}\right)\right\rangle}{\left\langle P_{z z}\left(z=z_{0}\right)\right\rangle} .
$$

The friction coefficient is evaluated at the center of the pore $\left(z=z_{0}\right)$, where the streaming velocity is zero. The point $z_{0}$ corresponds to the minima of the curves in Fig. 10. Values for $\xi^{*}\left(z_{0}^{*}\right)$ and other quantities are summarized in Table II. Results obtained at low grafting density by periodic, nonrandom assignment are also included in Table II for comparison. The limited set of dipole moments $\left\{\mu^{*}\right.$ 

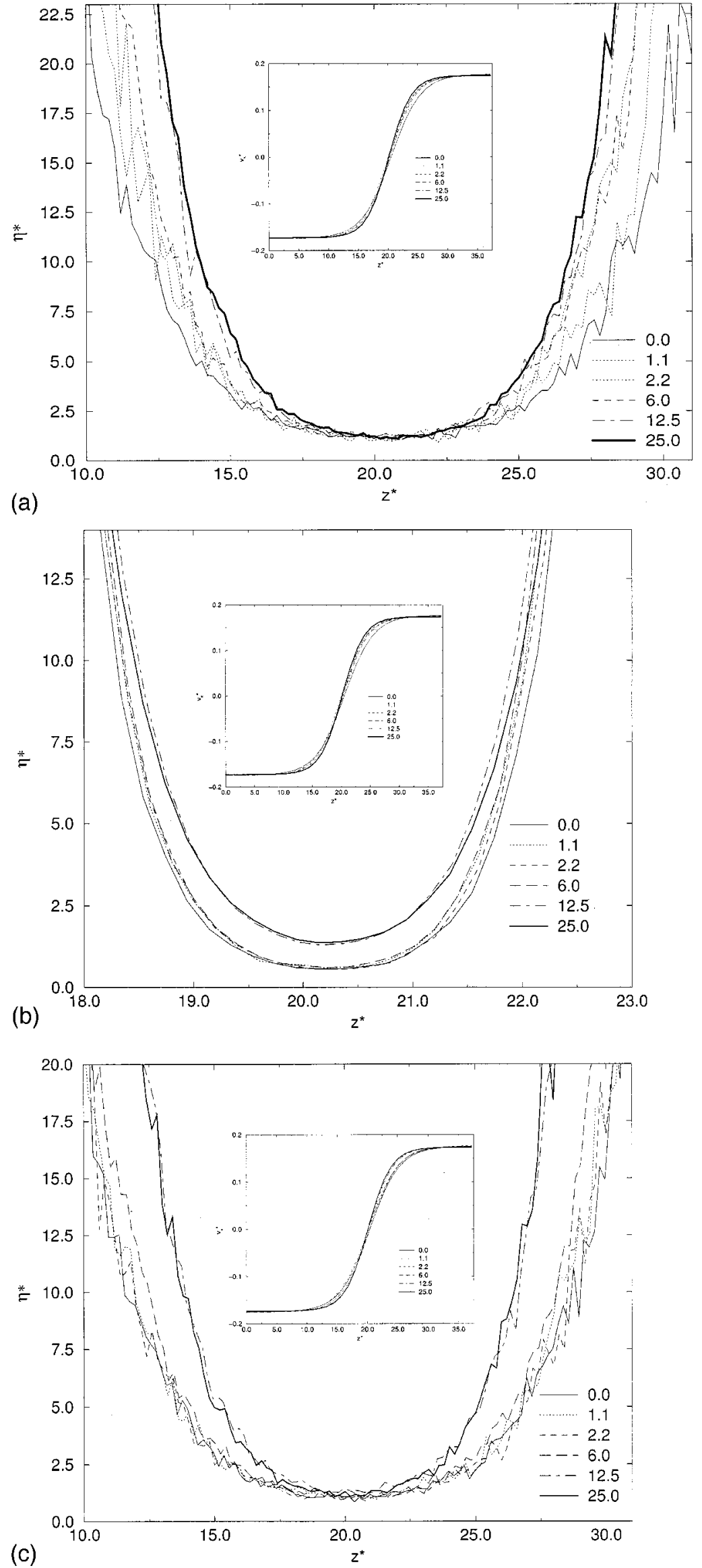

FIG. 10. Viscosity profiles $\eta^{*}\left(z^{*}\right)\left(\eta^{*}\right.$ $\left.=P / \dot{x}\left(M \sigma^{4} / \epsilon^{3}\right)^{1 / 2}\right)$ across the nanopore for different reduced dipole moments, $\mu^{*}$ (given in the inset). Only the central pore region is displayed (see Fig. 1). The profiles are calculated from Eq. (15) for the random configurations $R, 33 \%$ (a), $R$, $66 \%$ (b), and for the periodic, linked configuration $P, L$ (c). Note the different scales used on the axes. The corresponding velocity profiles $\dot{x}^{*}$ $\left(\dot{x}^{*}=\dot{x}(M / \epsilon)^{1 / 2}\right)$ are shown in the inset. 
TABLE II. Selected thermodynamic data determined at $\dot{x}^{*}\left(z^{*}=z_{0}^{*}\right)=0.0$, i.e., where the streaming velocity of the solvent is zero. All quantities are reported in reduced units, note, however that $\xi^{*}$ is dimensionless. $\eta^{*}\left(z_{0}^{*}\right)$ and $\xi^{*}\left(z_{0}^{*}\right)$ are friction and viscosity coefficients calculated from Eqs. (15) and (16), respectively. See Table I caption regarding the nomenclature of the configurations.

\begin{tabular}{|c|c|c|c|c|c|c|c|c|}
\hline Conf. & Cov. & $\mu^{*}$ & $T^{*}\left(z_{0}^{*}\right)$ & $\left(\frac{d \dot{x}\left(z^{*}\right)}{d z^{*}}\right)_{z^{*}=z_{0}^{*}}$ & $P_{x z}^{*}\left(z_{0}^{*}\right)$ & $P_{n}^{*}\left(z_{0}^{*}\right)$ & $\eta^{*}\left(z_{0}^{*}\right)$ & $\zeta^{*}\left(z_{0}^{*}\right)$ \\
\hline$R$ & 33 & 0.0 & 2.0530 & 0.0353 & -0.0428 & 10.7750 & 1.2119 & 0.00397 \\
\hline$R$ & 33 & 1.1 & 2.0670 & 0.0376 & -0.0420 & 10.7500 & 1.1168 & 0.00391 \\
\hline$R$ & 33 & 2.2 & 2.0380 & 0.0406 & -0.0420 & 10.7400 & 1.0337 & 0.00391 \\
\hline$R$ & 33 & 6.0 & 2.0560 & 0.0406 & -0.0472 & 10.7566 & 1.1618 & 0.00439 \\
\hline$R$ & 33 & 12.5 & 2.0544 & 0.0443 & -0.0592 & 10.7800 & 1.3359 & 0.00551 \\
\hline$R$ & 33 & 25.0 & 2.0540 & 0.0461 & -0.0530 & 10.7600 & 1.1488 & 0.00493 \\
\hline$R$ & 66 & 0.0 & 2.0588 & 0.1772 & -0.1012 & 4.7388 & 0.5627 & 0.02136 \\
\hline$R$ & 66 & 1.1 & 2.0520 & 0.1819 & -0.1132 & 4.7755 & 0.6115 & 0.02370 \\
\hline$R$ & 66 & 2.2 & 2.0462 & 0.1814 & -0.1038 & 4.8708 & 0.5597 & 0.02131 \\
\hline$R$ & 66 & 6.0 & 2.0650 & 0.1839 & -0.1159 & 5.2016 & 0.6194 & 0.02228 \\
\hline$R$ & 66 & 12.5 & 2.0744 & 0.1597 & -0.2116 & 5.7513 & 1.3077 & 0.03679 \\
\hline$R$ & 66 & 25.0 & 2.0738 & 0.1524 & -0.2118 & 5.7516 & 1.3769 & 0.03682 \\
\hline$P$ & 33 & 0.0 & 2.0593 & 0.0381 & -0.0417 & 10.8000 & 1.0934 & 0.00386 \\
\hline$P$ & 33 & 1.1 & 2.0420 & 0.0377 & -0.0412 & 10.7000 & 1.0903 & 0.00385 \\
\hline$P$ & 33 & 2.2 & 2.0590 & 0.0411 & -0.0443 & 10.7495 & 1.0777 & 0.00412 \\
\hline$P$ & 33 & 6.0 & 2.0535 & 0.0399 & -0.0465 & 10.7879 & 1.1657 & 0.00431 \\
\hline$P$ & 33 & 12.5 & 2.0500 & 0.0447 & -0.0577 & 10.7800 & 1.2892 & 0.00535 \\
\hline$P$ & 33 & 25.0 & 2.0485 & 0.0462 & -0.0555 & 10.7700 & 1.1989 & 0.00515 \\
\hline$P, L$ & 33 & 0.0 & 2.0480 & 0.0354 & -0.0378 & 10.7800 & 1.0667 & 0.00351 \\
\hline$P, L$ & 33 & 1.1 & 2.0500 & 0.0354 & -0.0373 & 10.6936 & 1.0534 & 0.00349 \\
\hline$P, L$ & 33 & 2.2 & 2.0537 & 0.0360 & -0.0356 & 10.7407 & 0.9869 & 0.00331 \\
\hline$P, L$ & 33 & 6.0 & 2.0520 & 0.0378 & -0.0421 & 10.7534 & 1.1114 & 0.00392 \\
\hline$P, L$ & 33 & 12.5 & 2.0579 & 0.0435 & -0.0592 & 10.7813 & 1.3598 & 0.00549 \\
\hline$P, L$ & 33 & 25.0 & 2.0490 & 0.0452 & -0.0534 & 10.7900 & 1.1787 & 0.00495 \\
\hline
\end{tabular}

$=0,1.1,2.2,6.0,12.5,25.0\}$ applied and summarized in Table II seems sufficient to capture upper and lower bounds of the variation of friction and viscosity data with magnitude of dipole moment for this model system. Calculations using more values of $\mu^{*}$ between 0 and 25 are not pursued, since they would probably not provide more insight into the rheological behavior of the grafted chains.

From Table II we conclude that the induced shear leads to almost identical temperatures in all systems. $T^{*}$ fluctuates around 2.04-2.07. Recall that all systems are Nosé-Hoover thermostated to 2.083 at the boundary walls. With respect to the friction forces at low grafting density, $\xi^{*}\left(z_{0}^{*}\right)$ reaches a maximum at $\mu^{*}=12.5$. We observe that almost identical frictions are determined for linked and unlinked systems. As indicated by the values of $\eta^{*}\left(z_{0}^{*}\right)$, the viscosity is also highest around $\mu^{*}=12.5$ at low grafting density. As shown in Figs. 6, 7, and 10 large differences occur in normal pressures and velocity gradients when comparing low and high grafting densities. Viscosity and friction data on the other hand appear quantitatively different. In particular for $\mu^{*} \leqslant 6.0$ one finds that the values for $\xi^{*}$ differ by almost an order of magnitude, whereas $\eta^{*}$ varies less.

At high grafting density and for the two largest dipolar strengths $\left(\mu^{*}=12.5\right.$ and $\left.\mu^{*}=25.0\right)$, viscosity and friction coefficients are approximately a factor of 2 higher than for the lower ones. This is mainly due to large differences in $\left\langle P_{x z}^{*}\left(z_{0}^{*}\right)\right\rangle$ as demonstrated when inspecting the pore regions of the curves in Figs. 8 and 9. The remarkably low friction and viscosity coefficients found for zero or small dipole moments essentially reflect the reduced number of solvent particles in the system causing $\left\langle P_{x z}^{*}\left(z_{0}^{*}\right)\right\rangle$ to become lower.

For the low grafting density, periodic and random grafting of the amphiphiles leads to practically identical viscosity and thermodynamical data indicating no significant sensitivity to the initial setup of the configuration of the grafted amphiphiles. The periodic, nonlinked configuration was generated as the linked configuration as outlined in Sec. II B. The only difference is that in these simulations, the potential covalently linking the two head groups, Eqs. (3) and (4), was not invoked.

Summarizing, we conclude that Table II demonstrates numerical differences between friction coefficients and viscosities at high and low grafting densities for all but the second highest dipole moment $\mu^{*}=12.5$. Chain linking does not affect these mechanical quantities. The difference in $\xi^{*}$ and $\eta^{*}$ observed for the two coverages is a combined effect due to differences in velocity gradients, due to differences in $\left\langle P_{x z}^{*}\left(z_{0}^{*}\right)\right\rangle$, and due to differences in $\left\langle P_{z z}^{*}\left(z_{0}^{*}\right)\right\rangle$. 
$\left\langle P_{z z}^{*}\left(z_{0}^{*}\right)\right\rangle,\left\langle P_{x z}^{*}\left(z_{0}^{*}\right)\right\rangle$ and the velocity gradient are all seen to vary significantly with grafting density. Within each of the two coverages, $\left\langle P_{x z}^{*}\left(z_{0}^{*}\right)\right\rangle$ displays the most pronounced dependence on the magnitude of $\mu^{*}$.

\section{CONCLUSION}

We have performed nonequilibrium molecular dynamics simulations to probe structural and dynamical properties of tail-grafted, amphiphilic chains under shear at different grafting densities. Emphasis was put on the modification of the head groups in order to investigate to which extent this modification of the interfacial region could specifically control the mechanical properties (reflected in friction and viscosity). Moreover, by considering two different grafting densities we were able to address the effect of dipolar forces across the nanopore with increasing load. The potentials describing the strength of the head-group interactions were chosen such that the corresponding variation in head-group type covers a wide range of amphiphilic molecules.

An experimental study addressing the effect of introducing dipolar head-group interactions to a confined liquid under shear was recently reported in Ref. [48]. Both ends of the polymers (tail and head-group) were functionalized by polar groups. Furthermore, not all of the chains were necessarily end grafted; some chains moved freely in the solvent. Accordingly the experimental study was carried out in the polymeric brush regime. The authors addressed two main questions. One regarding how the end-grafting property of the polymer depends on the chemical functionality of the end group, the other regarding how the molecular film responds to normal compression when interfacial dipolar forces are present. We emphasize that the systems utilized in the SFA work and our study are different in nature, since the studies address different questions. A comparison between the experimental data and our results is therefore yet not appropriate. However, combining the experimental methodology outlined in Refs. [23,48] with, e.g., the alkyl thiol monolayers introduced in Refs. [7,8] would be interesting, since this could in principle examine the essential findings of our study. The authors of Refs. [7,8] originally chose the variation in wettability as a macroscopic interfacial quantity to specifically probe the implications of a chemically manipulated interface, whereas we instead reported to probing the friction forces and viscosities. However, using, e.g., the model systems of Refs. [7,8] and invoking dielectric spectroscopy along with the SFA could possibly provide the experimental information on how dipolar forces are transmitted across interfaces and how they affect the shear rheology. Such an experiment could presumably examine to which extent macroscopic mechanical interfacial properties could be controlled by molecularly designing the interfaces. Supplementary information may be obtained by examining how the friction and viscosity of the supported lipid monolayer and bilayer used in Refs. $[9,10]$ are affected by introduction of dipoles.

Essentially the findings of the present study rely on a tunable repulsive dipolar interaction across the pore for a fixed monolayer separation of varying grafting density. The separation was intentionally chosen such that the interfaces should couple through dipolar interactions. Indeed this was observed; the coupling being strongest for the largest grafting density. Increasing the monolayer separation (via $L_{z}$, see Fig. 1) and keeping the number of beads in the amphiphiles fixed would lower friction and viscosity for both grafting densities. The limit of uncoupling would be approached essentially as $1 / \bar{r}^{3}$, where $\bar{r}$ is the average distance of two interacting dipoles residing in the two opposing monolayers.

In the chosen coupling regime, our model systems show significant differences in the viscosity data and hence in friction coefficients when comparing high and low grafting densities and in particular when comparing low $\left(\mu^{*}\right.$ $=0.0-6.0)$ and high $\left(\mu^{*}=12.5-25.0\right)$ dipole moments. In the interfacial region, the viscosity data are strongly dependent on dipolar interactions. At intermediate to high dipole moments the amphiphile-solvent interface is less diffusive when compared to small or no dipole moments. At high grafting density the viscosity in the solvent region increases with increasing dipolar repulsion. The friction coefficient is found to be remarkably small when no or only small dipoles are applied. Surprisingly, chain linking (i.e., constraining the head-group movement) has essentially little or no effect on the shear rheology. On the basis of our observations one would therefore not expect any significant differences in the flow characteristics between a tail-grafted system of crosslinked and nonlinked amphiphiles within each monolayer. The dipolar effects are far more pronounced. Differences found in viscosities and friction coefficients within the same grafting density are predominantly assigned to a larger response of the normal pressure to the applied shear field; i.e., due to a larger configurational contribution to the response component $\left\langle P_{x z}(z)\right\rangle$. At high grafting density, the normal pressure $\left\langle P_{z z}(z)\right\rangle$ is strongly affected by the magnitude of dipolar repulsion. Velocity gradients are very sensitive to the grafting density, but less affected by the modifications of the head groups. Increasing dipolar interactions broadens the solvent distributions leading to a wider and less diffusive amphiphile-solvent interface. In contrast, we find in the absence of dipoles that the solvent molecules essentially are expelled from the boundary zones toward the central pore region. This displacement generates a more diffusive interface and thereby lowers friction and viscosity. The geometric data of the amphiphiles are characteristically different for the two grafting densities. At large density, chain folding and entanglement are reduced. In contrast, dipolar repulsion promotes chain folding and/or entanglement. The overall result is that interfacial viscosities and friction forces increase in tail-grafted amphiphilic systems where repulsive head-group interactions are significant, i.e., where strong coupling between the interfaces occur.

\section{ACKNOWLEDGMENTS}

Financial supports from the European Grant EU No. BIO 4 CT 972365 and from The Danish Natural Science and Technical Science Research Councils are gratefully acknowledged. The MEMPHYS group was supported by the Hasselblad Foundation. 
[1] D. J. Burgess and N. O. Sahin, J. Colloid Interface Sci. 189, 74 (1997).

[2] J. E. Valentini et al., Ind. Eng. Chem. Res. 35, 434 (1996).

[3] S. M. Murray and E. Dickinson, Food Sci. Technol. Int. 2, 131 (1996).

[4] D. C. Clark et al., in Food Colloids and Polymers, Stability and Mechanical Properties, Royal Society of Chemistry Special Publ. No 113, edited by E. Dickinson and P. Walstra (Royal Society of Chemistry, Cambridge, 1993), p. 354.

[5] D. C. Clark, A. R. Mackie, P. J. Wilde, and D. R. Wilson in Food Proteins-Structure and Functionality, edited by K. D. Schwenke and R. W. Mothes (VCH, Berlin, 1993), p. 263.

[6] B. Warburton, Curr. Opin. Colloid Interface Sci. 1, 481 (1996).

[7] C. D. Bain and G. M. Whitesides, Angew. Chem. Int. Ed. Engl. 28, 506 (1989).

[8] C. D. Bain, J. Evall, and G. M. Whitesides, J. Am. Chem. Soc. 111, 7155 (1989).

[9] N. Boden et al., Tetrahedron 54, 11537 (1998).

[10] J. Sagiv, J. Am. Chem. Soc. 102, 92 (1908).

[11] V. A. Parsegian and E. A. Evans, Curr. Opin. Colloid Interface Sci. 1, 53 (1996).

[12] D. A. Hammer and M. Tirrell, Annu. Rev. Mater. Sci. 26, 651 (1996).

[13] D. Leckband, Biophys. J. 68, 2215 (1996).

[14] N. Dan, Curr. Opin. Colloid Interface Sci. 1, 48 (1996).

[15] S. S. Patel and M. Tirrell, Annu. Rev. Phys. Chem. 40, 597 (1989).

[16] H. J. Taunton, C. Toprakcioglu, L. J. Fetters, and J. Klein, Nature (London) 332, 712 (1988).

[17] H. J. Taunton, C. Toprakcioglu, L. J. Fetters, and J. Klein, Macromolecules 23, 571 (1990).

[18] S. Granick, Science 253, 1374 (1991).

[19] J. Klein et al., Nature (London) 370, 634 (1994).

[20] J. Klein, E. Kumacheva, D. Perahia, D. Mahalu, and S. Warburg, Faraday Discuss. 98, 173 (1994).

[21] B. Bushhan, J. N. Israelachvili, and U. Landman, Nature (London) 374, 607 (1995).

[22] S. S. Patel and M. Tirrell, Annu. Rev. Phys. Chem. 2, 271 (1997).

[23] Y. K. Cho, H. Watanabe, and S. Granick, J. Chem. Phys. 110, 5532 (1999).

[24] G. S. Grest, J. Chem. Phys. 105, 5532 (1996).
[25] G. S. Grest, Phys. Rev. Lett. 76, 4979 (1996).

[26] M. J. Stevens, M. Mondello, and G. S. Grest, J. Chem. Phys. 106, 7303 (1997).

[27] I. M. Neelov, O. V. Borisov, and K. Binder, J. Chem. Phys. 108, 6973 (1998).

[28] L. Miao, H. Guo, and M. J. Zuckermann, Macromolecules 29, 2289 (1996).

[29] P.-Y. Lai and K. Binder, J. Chem. Phys. 98, 2366 (1993).

[30] G. S. Grest, Curr. Opin. Colloid Interface Sci. 2, 271 (1997).

[31] G. H. Peters and D. J. Tildesley, Phys. Rev. E 2, 1182 (1995).

[32] G. H. Peters and D. J. Tildesley, Phys. Rev. E 5, 5493 (1996).

[33] M. L. Gee, P. M. McGuiggan, J. Israelachvili, and A. M. Homola, J. Chem. Phys. 93, 1895 (1990).

[34] J. D. Weeks, D. Chandler, and H. C. Andersen, J. Chem. Phys. 54, 5237 (1971).

[35] K. Kremer and G. S. Grest, J. Chem. Phys. 92, 5057 (1990).

[36] K. Kremer, in Computer Simulation in Chemical Physics, Vol. 397 of NATO Advanced Study Institute, Series C: Mathematical and Physical Science, edited by M. Allen and D. J. Tildesley (Kluwer Academic, London, 1993).

[37] S. Y. Liem, D. Brown, and J. H. R. Clarke, Phys. Rev. A 45, 3706 (1992).

[38] D. A. Pink, M. Belaya, V. Levadny, and B. Quinn, Langmuir 13, 9327 (1997).

[39] M. P. Allen and D. J. Tildesley, Computer Simulation of Liquids (Clarendon, Oxford, 1988).

[40] P. J. Flory, Statistical Mechanics of Chain Molecules (Interscience, New York, 1996).

[41] Y. Liem, Ph.D. thesis, University of Manchester, Manchester, United Kingdom, 1992.

[42] D. H. Tsai, J. Chem. Phys. 70, 1375 (1979).

[43] J. P. R. B. Walton, D. J. Tildesley, and J. S. Rowlinson, Mol. Phys. 48, 1357 (1983).

[44] B. D. Todd, D. Evans, and P. Daivis, Phys. Rev. E 52, 1627 (1995).

[45] F. Varnik, J. Baschnagel, and K. Binder, J. Chem. Phys. 113, 4444 (2000).

[46] J. P. R. B. Walton, D. J. Tildesley, and J. S. Rowlinson, Mol. Phys. 58, 1013(E) (1986).

[47] H. A. Barnes, J. F. Hutton, and K. Walters, An Introduction to Rheology (Elsevier, Amsterdam, 1989).

[48] M. Ruths and S. Granick, J. Phys. Chem. B 103, 8711 (1999). 